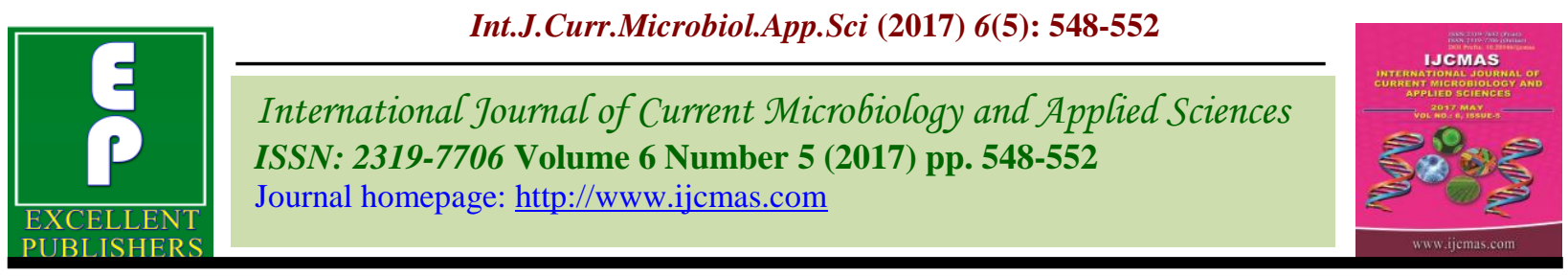

Original Research Article https://doi.org/10.20546/ijcmas.2017.605.063

\title{
Varying Drug Resistant Patterns of MRSA Isolates with PVL Gene
}

\author{
Sujitha Elan Seralathan $^{1 *}$, C. Sheeladevi ${ }^{1}$, N. Shashikala ${ }^{1}$, Saranathan $^{2}$ and K. Prashanth ${ }^{2}$ \\ ${ }^{1}$ Department of Microbiology Pondicherry Institute of Medical Sciences, Pondicherry Institute of \\ Medical Sciences, Pondicherry, India \\ ${ }^{2}$ Department of Biotechnology, Pondicherry University, Pondicherry, India \\ *Corresponding author:
}

\section{A B S T R A C T}

Nosocomial infections due to Methicillin Resistant Staphylococcus aureus (MRSA) has been an element of concern to the medical personnel for the past four decades. Community associated MRSA (CA-MRSA) which was initially considered as more sensitive than hospital acquired MRSA (HA-MRSA) is now presenting with increasing levels of drug resistance. Along with the mecA gene, pvl gene is characteristically present in most isolates of CA-MRSA. To study the varying drug resistant patterns of MRSA isolates with pvl gene. A total of 150 clinical isolates of MRSA analysed in the study were subjected to susceptibility testing to cefoxitin (30 Â $\mu$ g) and growth on oxacillin screen agar containing $6 \hat{\mathrm{A}} \mu \mathrm{g} / \mathrm{mL}$ of oxacillin for the detection of methicillin resistance. All the isolates which were included in the study, were checked by PCR for the presence of mecA gene, which codes for methicillin resistance and for pvl gene. Amplification of 540bp and 625bp gene fragments in the PCR reaction indicates the presence of mecA and pvl genes respectively. mecA gene was present in all the 150 isolates of MRSA and pvl gene was present only in 26 isolates. Of these 26 isolates that had pvl gene, 14 were in MRSA isolated from outpatient samples and were sensitive to most of the non- beta lactam antibiotics. Among the other 12 inpatient MRSA isolates which had the pvl gene, nine were sensitive to most of the non- beta lactam antibiotics, whereas remaining three were resistant to most of the antibiotics except vancomycin and linezolid. The presence of pvl gene can no longer be used to discriminate between CA-MRSA and HA-MRSA. Indiscriminate empirical treatment of MRSA infections with high end antibiotics like glycopeptides needs to avoided and therapy with non beta lactam antibiotics like lincosamides which have better soft tissue penetration should be used as very few new antimicrobial agents are in the pipeline.

\section{Introduction}

Traditionally MRSA has been considered as a major nosocomial pathogen in health care facilities, but in the last decade it has also been observed to be an emerging pathogen causing increasing number of community acquired infections. The emergence of this pathogen depends on its ability to survive in different environments and to interact successfully with the host. It was initially presumed that community acquired MRSA infection is attributed to the presence of Panton Valantine Leucocidin $(P V L)$ toxin encoded by $p v l$ gene. However, the presence of $p v l$ gene alone cannot be considered as a 
marker of CA-MRSA (Vandenesch et al., 2003; Shenoy et al., 2010). The community acquired strains tend to be more susceptible to non- beta lactam agents as compared to hospital acquired MRSA isolates and appear to carry a unique Staphylococcal chromosome (SCC mec type IV) in relation to resistant genes.

MRSA are highly virulent strains capable of clonal dissemination and have the ability to cause epidemics of furunculosis and other skin and soft tissue infections, irrespective of characteristics of populations or the health care setting (Rachel, 2008; Harbarth et al., 2005). The MRSA isolates carrying the $P V L$ toxin possesses a serious threat and is a major public health concern (Vandenesch et al., 2003; Shenoy et al., 2010). The aim of the present study was to describe the varying drug resistant patterns of MRSA isolates with $p v l$ gene.

\section{Methods}

This prospective study was carried out in the Department of Clinical Microbiology, Pondicherry Institute of Medical Sciences (PIMS). All the clinically significant 150 MRSA isolates were included in the study.

The clinical isolates were screened for methicillin resistance by susceptibility to cefoxitin $(30 \mu \mathrm{g})$ and growth on oxacillin screen agar containing $6 \mu \mathrm{g} / \mathrm{mL}$ of oxacillin. Antibiotic susceptibility testing was done on Muller Hinton agar by Kirby Bauer disc diffusion method for the following antibiotics; Trimethoprin-Sulphamethoxazole (1.25/23.75 $\mu \mathrm{g})$, Tetracycline $(30 \mu \mathrm{g})$, Linezolid $(30 \mu \mathrm{g})$, Erythromycin $(15 \mu \mathrm{g})$, Clindamycin $(2 \mu \mathrm{g})$, Teicoplanin $(30 \mu \mathrm{g})$, Chloramphenicol (30 $\mu \mathrm{g})$, Ciprofloxacin $(5 \mu \mathrm{g})$, Penicillin (10 units), Amikacin $(30 \mu \mathrm{g})$ and Gentamicin (10 $\mu \mathrm{g}$ ) (Hi-media) were recorded as per CLSI guidelines. The MIC for vancomycin against the 150 MRSA Isolates was determined by agar dilution method.

\section{Molecular Methods for MRSA detection}

mecA gene, which codes for Methicillin resistance and $p v l$ gene were detected using PCR for all the 150 MRSA isolates included in the study. The following primers procured from Hi-media (India) were used in the study.

mecA (forward primer $1 \mu \mathrm{L} ; 5$ ' GTA GAA ATG ACT GAA CGT CCG ATAA 3'), (Reverse primer $1 \mu 1$; 5' CCA ATT CCA CAT TGT TTC GGT CTAA 3').

Pvlgene (forward primer $1 \mu \mathrm{l}$; 5'ATCATTAGGTAAAATGTCTGGACATG ATCC A-3'), (Reverse primer $1 \mu \mathrm{l}$; 5'GCATC AST GTA TTG GAT AGC AAA AGC - 3').

The amplified products were analysed by agarose gel electrophoresis. Amplification of $540 \mathrm{bp}$ and 625bp gene fragments in the PCR reaction indicates the presence of mecA and $p v l$ genes respectively. The positive control strain used in PCR for mecA was ATCC 43300 and the negative control was ATCC 25973.

Clinical details of the patient and the demographic information, history of antibiotic usage, previous hospitalization, comorbidities like Diabetes, post surgical status were recorded in a designed proforma. The patients from whom MRSA was isolated were followed up and the responses to treatment were noted.

\section{Results and Discussion}

A total of 354 Staphylococcus aureus were isolated out of which 150 were found to be MRSA. Rate of methicillin resistance among the Staphylococcus aureus isolates was $42.37 \%$. Among the 150 patients from whom MRSA was isolated, 68 patients (45.67\%) had a prolonged hospital stay of greater than 7 
days duration, 62 patients $(41.25 \%)$ had surgical interventions, 59 patients $(39.6 \%)$ were referred from other hospitals and 39 patients $(26.1 \%)$ were admitted in ICU for more than 2 days. Isolates from exudates which included pus, wound swab, and sterile body fluids (apart from blood) constituted $90.67 \% \quad(n=136)$ of isolates. Clinically significant respiratory isolates were from tracheal aspirate and bronchoalveolar lavage which was found to be $7.3 \%(n=11)$. Two isolates were from blood and only one isolate was from urine (Figure1).

$m e c A$ gene was present in all the 150 isolates of MRSA and $p v l$ gene was present only in 26 isolates. All the $26 \mathrm{MRSA}$ isolates with $p v l$ gene were from patients with skin and soft tissue infection. The MRSA isolates from blood and other sterile sites did not carry the $p v l$ gene. Of the 26 isolates which had $p v l$ gene, 14 were from MRSA isolated from outpatient samples and were sensitive to most of the non- beta lactam antibiotics and 12 were from MRSA isolated from inpatient samples. Among these 12 inpatient MRSA isolates which had the $p v l$ gene, nine were sensitive to most of the non- beta lactam antibiotics, whereas remaining three isolates were resistant to the non beta lactam antibiotics like quinolones,macrolides, lincosamide and tetracycline except vancomycin and linezolid. Multidrug resistant MRSA strains were detected in the study. Only three isolates showed multidrug resistance, two of them exhibited inducible clindamycin resistance and one of them exhibited constitutive resistance to clindamycin.

Among these three isolates, one of them was from the wound swab of a patient with diabetic foot and the other two were from gluteal abscess pus and post auricular abscess. Following the culture report they were started on oral linezolid to which they responded well.
Historically, infections caused by methicillinresistant Staphylococcus aureus (MRSA) were predominantly associated with patients in hospitals and skilled nursing facilities. In recent years, reports of community-associated MRSA infections (CA-MRSA) have been increasing (Vandenesch et al., 2003; Shenoy et al., 2010). Several studies conducted in India and elsewhere have shown that the prevalence of CA-MRSA to be varied ranging from 15 to $32 \%$ (Vandenesch et al., 2003; Shenoy et al., 2010). A significant difference has been demonstrated in the susceptibility of CA-MRSA and HA-MRSA by various investigators (Hacek et al., 2009; Diep et al., 2006; Ruhe et al., 2007). Hospital acquired MRSA (HA-MRSA) have been found to exhibit resistance to frequently used non betalactam antibiotics whereas Community acquired MRSA (CA-MRSA) are known to cause skin and soft tissue infections and are usually susceptible to lincosamides like clindamycin which have better tissue penetration and absorption.

Diverse spectrum Studies by Ruhe and Menon have suggested that non beta-lactam antibiotics like tetracycline, which can be administered orally as a feasible option for treatment of CA-MRSA infection (Ruhe et al., 2007). However, few strains of CAMRSA have shown to exhibit multi drug resistance to most of the commonly used non beta lactam antibiotics. The incidence of CAMRSA was $17.33 \%(n=26 / 150)$ in the present study which is in concurrence with the findings of various other studies. However, these investigators have studied the incidence of CA-MRSA in the community. The incidence of CA-MRSA $i$ in a tertiary care centre, as studied by Kanerva et al., (2009) is found to be $21 \%$. Most of the CA-MRSA isolates $(88 \%)$ were primarily found to be susceptible to antibiotics like clindamycin, amikacin, erythromycin, cotrimoxazole, tetracycline and chloramphenicol, unlike HAMRSA. 
A study done by Nandita et al., 2016 have shown an increased rate of susceptibility to clindamycin $(84 \%)$ and have recommended the presence of $p v l$ gene along with clindamycin susceptibility to be a predictor to detect community origin of MRSA (Nandita et al., 2016). In our study also, it was found that the $88.5 \%$ of isolates carrying the $p v l$ gene and susceptible to clindamycin was found to be statistically significant $(\mathrm{p}<0.001)$. Clindamycin has an excellent tissue penetration and is effective for the treatment of skin and soft tissue infections (Mandelia $e t$ al., 2010).

Fig.1 Distribution of the isolates of MRSA

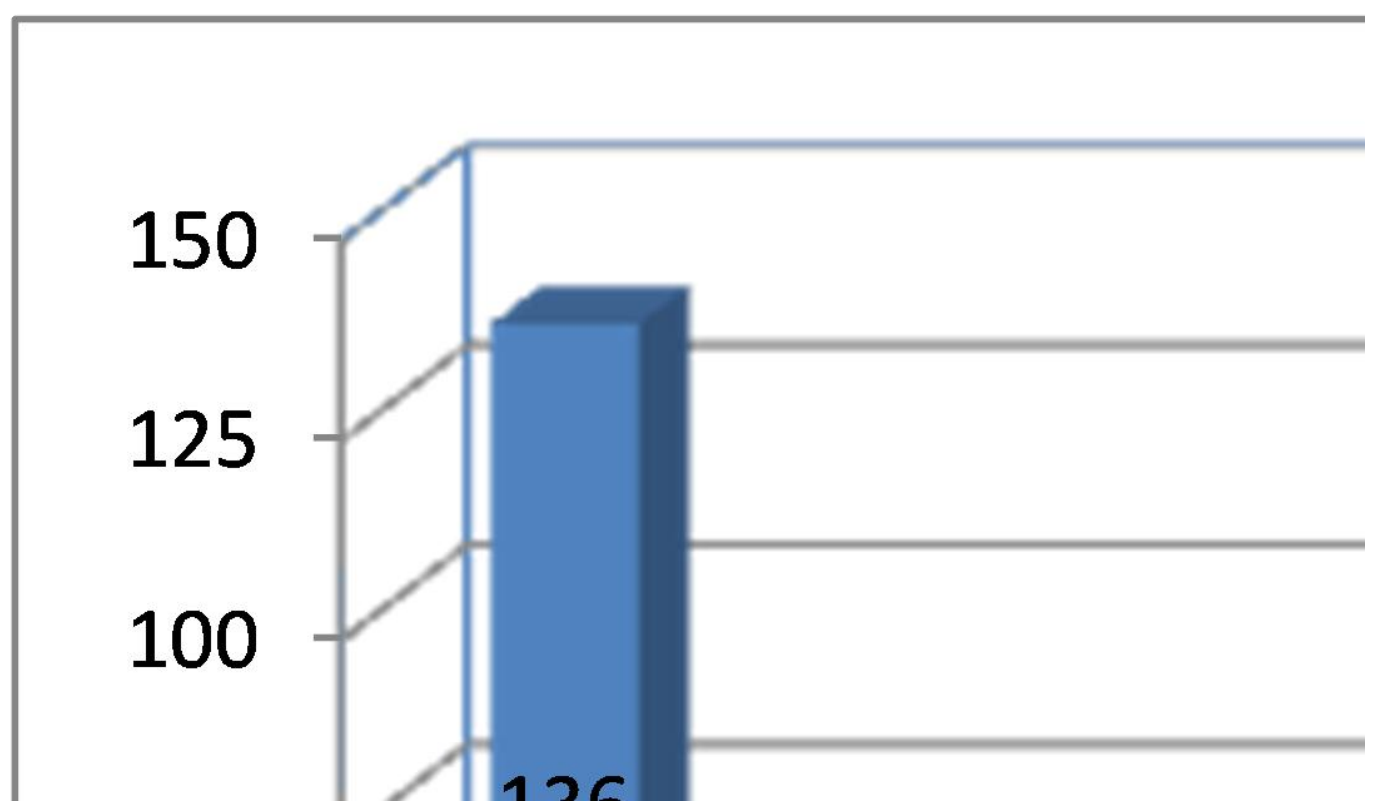

It has been reported that the action of $p v l$ toxin in the tissue is decreased even by sub inhibitory concentration of clindamycin (Dumitrescu et al., 2007). However, three among the 26 CA-MRSA isolates showed multi drug resistance and were sensitive only to vancomycin and linezolid. Among the MRSA isolates carrying the $p v l$ gene, two of them exhibited inducible clindamycin resistance which is in contrast to Vysakh et $a l$., wherein they reported that $44.4 \%$ of their MRSA isolates carrying the $p v l$ gene showed inducible clindamycin resistance (Vysakh et al., 2013).

Emergence of multi drug resistance among CA-MRSA isolates is of major concern to the society. This is attributed to the indiscriminate empirical use of high end antibiotics like vancomycin and linezolid by many clinicians without using other non beta lactum antibiotoics as the first line of management. In our study, the prevalence of CA-MRSA with $p v l$ gene positive isolates was $17.33 \%$. Studies on prevalence of $p v l$ gene in various geographical settings have shown varied results, where the prevalence varies from 13.6 to $21.7 \%$. The presence of $p v l$ gene can no longer be used to discriminate between CAMRSA and HA-MRSA. However, MRSA isolates carrying the pvl gene are predominantly associated with skin and soft tissue infections. Clindamycin, which is a lincosamide has an excellent skin and soft tissue penetration and can be a potent choice to treat infections caused by strains of MRSA carrying the pvl gene.

Prevention of CA-MRSA is of utmost importance in health care setting ${ }^{(14)}$. Simple 
techniques like hand washing, barrier nursing and screening of patients for MRSA referred from other healthcare centres prior to admission for MRSA provide a long way for controlling the transmission of CA-MRSA infection.

\section{References}

Bhutia, K.O., T. Singh. 2012. The prevalence and the risk factors, which are, associated with Staphylococcus aureus and methicillinresistant Staphylococcus aureus which harboured the Panton ValentineLeukocidin gene in Sikkim. J. Clin. Diagn. Res., 6(3): 393-99.

Diep, et al. 2006. Prevalence of Inducible Clindamycin Resistance among CA-MRSA HA-MRSA Staphylococcus aureus isolates. J. Clin. Microbiol., 34: 2623-25.

Dumitrescu, O., Boisset, S., Badiou, C., Bes, M., Benito, Y., Reverdy, M.E., et al. 2007. Effect of Antibiotics on Staphylococcus aureus Producing Panton-Valentine Leukocidin. Antimicrob Agents Chemother., 51(4): 1515-19.

Hacek, D.M., Suzanne, M., Paule, Thomson, R.B., Jr., Robicsek, A. and Peterson, L.R. 2009. Implementation of Universal admission surveillance and Decolonization Program for methicillin-resistant Staphylococcus aureus (MRSA) reduces the number of MRSA and total number of S.aureus isolates reported by the clinical laboratory. J. Clin. Microbiol., 47: 3749-52.

Han, et al. 2007. Prevalence of Inducible Clindamycin Resistance among CAMRSA. J. Clin. Micro., 39: 1818-22.

Harbarth, S., et al. 2005. Community associated methicillin resistant Staphylococcus aureus, Emer. Infect. Dis., Switzerland 11(6): 962965.
Kanerva, M., et al. 2006. Community-associated methicillin-resistant Staphylococcus aureus isolated in Finland in 2004 to 2006. J. Clin. Microbiol., 47: 2655-7.

Mandelia, C., Shenoy, S. 2010. Community Associated-Methicillin-resistant Staphylococcus aureus in skin and soft tissue infections. J. Clin. Diagn. Res., 4: 2673-77.

Nandita, S., Niveditha, N., D.M. Thappa, S. Sistla. 2016. Can Panton Valentine Leukocidin Gene and Clindamycin Susceptibility Serve As Predictors of Community Origin of MrsaFrom Skin and Soft Tissue Infections? J. Clin. Diag. Res., Vol-10(1): DC01-DC04

Rachel, G. 2008. Community associated methicillin resistant Staphylococcus aureus: Epideimiology and update, J. Pediatric Infectious Dis., 27(10): 925-6

Ruhe, J.J. and Menon, A. 2007. Tetracyclines as an oral Treatment Option for patients with Community onset Skin and soft Tissue infections caused by Methicillin-Resistant Staphylococcus aureus. J. Antimicrobial Agents and Chemother., 3258-3303.

Shenoy, M.S., et al. 2010. Significance of MRSA strains in community associated skin and soft tissue infections, Ind. J. Med. Microbiol., 28(2): 152-4.

Vandenesch, F., Naimi, T., Enright, M.C., Lina, G., Nimmo, G.R., Heffernan, H., et al. 2003. Community acquired methicillin resistant Staphylococcus aureus carrying the Panton Valentine Leukocidin genes: Worldwide Emergence Emerging Infect. Dis., 9(8): 978-84.

Vysakh, P.R., Jeya, M. 2013. A comparative analysis of community acquired and hospital acquired methicillin-resistant Staphylococcus aureus. J. Clin. Diagn. Res., 7(7): 1339-42.

\section{How to cite this article:}

Sujitha Elan Seralathan, C. Sheeladevi, N. Shashikala, Saranathan and Prashanth, K. 2017. Varying Drug Resistant Patterns of MRSA Isolates with PVL Gene. Int.J.Curr.Microbiol.App.Sci. 6(5): 548-552. doi: https://doi.org/10.20546/ijcmas.2017.605.063 